\title{
Actividad espontánea de nociceptores cutáneos en pacientes con neuropatía de fibras delgadas
}

\author{
MARIO CAMPERO ${ }^{1,2}$, SEBASTIÁN CAMPERO $^{\text {a }}$
}

\section{Spontaneous activity of cutaneous nociceptors in patients with painful polyneuropathy. Report of three patients}

Painful polyneuropathy may result from selective impairment of small diameter nerve fibers, while tactile and motor functions are preserved. In these patients clinical and electrophysiological assessment is usually unrevealing. We report three patients with a pure painful polyneuropathy. One of them had neurogenic pruritus additionally. Quantitative sensory analysis disclosed a slight warm hypoesthesia (3/3) and paradoxical hot sensation (2/3) in the feet. Intraneural recordings from the peroneal nerve demonstrated abnormal spontaneous activity in 8 of 17 nociceptive afferents. One of them displayed double firing reflecting impulse multiplication. These results support the notion that patients with pain or pruritus with a distal distribution similar to a polyneuropathy, could have small diameter afferent fiber damage, despite normal function of large diameter fibers.

(Rev Med Chile 2012; 140: 1383-1390).

Key words: Ectopic activity; Neuralgia; Nociceptors; Polyneuropaties.

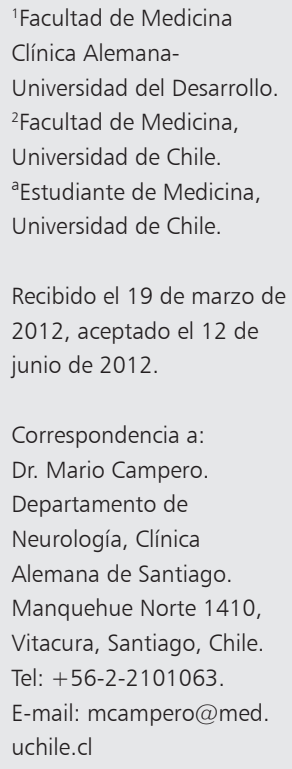

L a polineuropatía es una afección frecuente de nervios periféricos que da cuenta de síntomas y signos deficitarios (negativos), y positivos; en este último caso por exceso de función. Entre los primeros, se encuentran la hipoestesia a las diferentes modalidades (v.g. tibieza, frío, tacto, dolor), paresia y atrofia, y anhidrosis o hipotermia por hipersensibilidad por denervación ${ }^{1}$. Los síntomas positivos dan cuenta de parestesias (espontáneas), disestesias (sensación no dolorosa inducida por estímulo) y dolor e hiperalgesia. Las polineuropatías dolorosas comprometen fibras delgadas (mielínicas A-delta y amielínicas C) con o sin compromiso adicional de fibras gruesas (A-beta). Así, un paciente se puede presentar sólo con dolor e hiperalgesia con una distribución en calcetín, en ausencia de hipostesia o síntomas motores. En estos pacientes los reflejos osteotendinosos son normales lo mismo que los estudios convencionales -electromiografía y velocidad de conducción nerviosa-, generando una dificultad en el diagnóstico². El apoyo de los estudios cuantitativos somatosensoriales, en especial el análisis térmico somatosensorial (ATS) son de utilidad, a pesar de su condición psicofísica. Estos exámenes permiten determinar los umbrales de frío y tibieza así como de dolor térmico en las regiones sintomáticas y señalan hipoestesia e hipo o hiperalgesia térmica. Si bien estos estudios aportan significativamente al diagnóstico de neuropatía de este tipo de fibras, no hay una evidencia que demuestre directamente la alteración de las fibras delgadas que den cuenta de los síntomas. En este trabajo se presentan 3 pacientes con la sospecha de una polineuropatía de fibras delgadas en los que clínica y electrofisiológicamente no había compromiso de fibras gruesas y el diagnóstico se había puesto en duda. Se describe la presencia de actividad eléctrica anormal en nociceptores cutáneos que, con toda probabilidad, dan cuenta de los síntomas sensitivos positivos en los pacientes. 


\section{Métodos}

Se presentan 3 pacientes con diagnóstico de una polineuropatía dolorosa. Los 3 individuos tuvieron un examen neurológico completo, con énfasis en la sensibilidad. Se les realizó un estudio electrodiagnóstico (Nicolet Viking Quest) con técnica estándar de conducción nerviosa y electromiografía de aguja ${ }^{3}$. En todos se obtuvieron los umbrales de sensibilidad térmica mediante un estudio de AST (Medoc, Israel), como se detalla en otros artículos ${ }^{4}$. Un termode de $9 \mathrm{~cm}^{2}$ operando en el principio de Peltier es enfriado o entibiado de acuerdo a la dirección de la corriente que circula entre sus conductores, y la pendiente de enfriamiento o calentamiento, controlada por la cantidad de corriente. El operador controla un botón que inicia el cambio térmico desde una temperatura basal de $32^{\circ} \mathrm{C}$ a razón de $0,5^{\circ} \mathrm{C} / \mathrm{s}$ (para la sensación térmica) o $2^{\circ} \mathrm{C} / \mathrm{s}$ (para la sensación de dolor térmico) con un límite de $50^{\circ} \mathrm{C} \mathrm{y} 0^{\circ} \mathrm{C}$. El paciente debe señalar la primera sensación térmica o de dolor térmico oprimiendo un botón que revierte la temperatura hacia la basal. La sensación de frío, tibieza, dolor frío y dolor caliente se computan como el promedio de 4 estímulos consecutivos separados por $10 \mathrm{~s}$ de reposo en la temperatura neutra. Respecto a la microneurografía, permite registrar la actividad eléctrica de un número reducido de fibras aferentes de pequeño diámetro que median las sensaciones térmicas inocuas y nociceptivas. Si bien la técnica ha sido descrita con detalle ${ }^{5,6}$, daremos aquí una breve sinopsis de esta. En un paciente despierto se introduce un electrodo de tungsteno a través de la piel hasta contactar un nervio superficial que proyecte a la zona sintomática. La punta desnuda del electrodo mide $5 \mu$, mientras que la parte más gruesa de este tiene un diámetro de $200 \mu$, con una impedancia nominal de $1 \mathrm{M} \Omega$. Un electrodo de muy baja impedancia se inserta en el tejido subcutáneo a 1-2 cm del de registro. Ambos electrodos alimentan un preamplificador diferencial cuya señal es amplificada X 2.000 a 10.0000 veces y filtrada con una ventana entre 2,5 y $3.000 \mathrm{~Hz}$ (FHC Cell X3, Bowdowin, USA). La señal luego pasa por un sistema de eliminación de ruido (Humbug, Quest Scientific, Canada) y es luego digitalizada (NI USB 6100) y almacenada en un PC. La señal digitalizada es analizada 'on y off-
Figura 1. Umbrales de sensibilidad térmica medidos en la región tarsal con el método de límites. El umbral se expresa como la media de 3 estímulos sucesivos con $10 \mathrm{~s}$ entre ellos. A. Umbrales térmicos en el paciente 2, destacando un umbral para la sensación de frío normal y una marcada hipoestesia a la tibieza. B. En contraste, un sujeto normal de la misma edad que el paciente, tiene un umbral de tibieza de $34,5^{\circ} \mathrm{C}$.

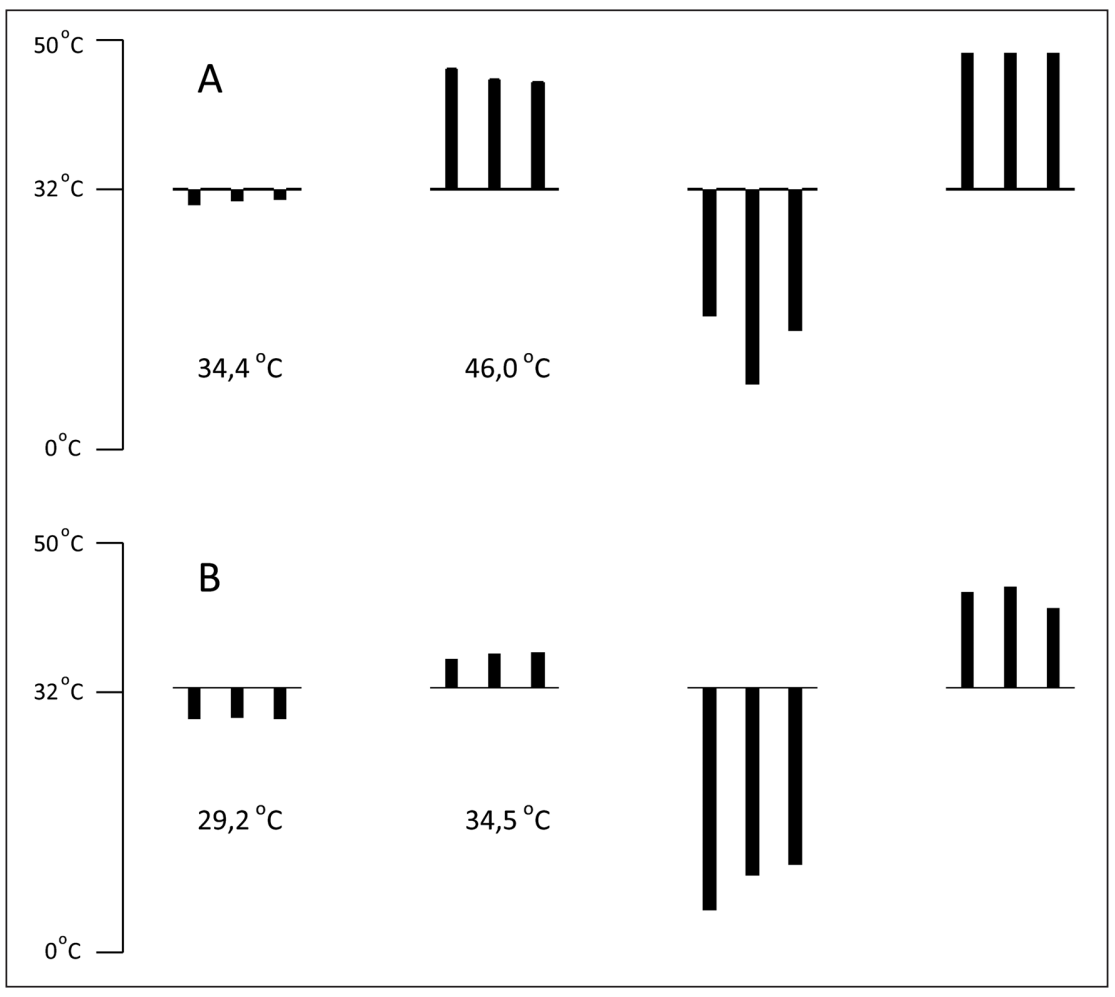


line' mediante el software QTRACW (copyright Institute of Neurology, Londres).

Los datos se grafican en la forma de muestra de puntos, en donde cada punto representa las mayores amplitudes en un barrido de $850 \mathrm{~ms}$ cada uno (Figura 2), disparado por un estímulo eléctrico en el campo receptivo cutáneo del fascículo desde el cual se registra. La identificación del tipo de aferente se logra mediante la aplicación de un protocolo de estimulación eléctrica del campo receptivo de una unidad con una frecuencia basal de $0,25 \mathrm{~Hz}$, la que se ve interrumpida por 3 min de estimulación a baja frecuencia $(1 / 30 \mathrm{~s}$; 'pausa') y por un tétano de $2 \mathrm{~Hz}$ también por 3 min. Durante la pausa, el axón normalmente se repolariza y recupera su latencia basal más rápida, enlenteciéndose luego del inicio de la frecuencia basal. Durante el tétano la latencia aumenta considerablemente, recuperando su latencia basal una vez finalizado este. Se han podido distinguir 4 patrones de cambios de latencia con este protoco$10^{7-9}$ : el tipo 1 , en que la fibra amielínica aumenta progresivamente la latencia más de $10 \%$ durante el tétano, y corresponden a nociceptores. Hay 2 subtipos: aquellos que mantienen su latencia durante la pausa (tipo 1a) y que corresponden a los nociceptores polimodales, sensibles al estímulo mecánico, térmico y químico, y los que recupera

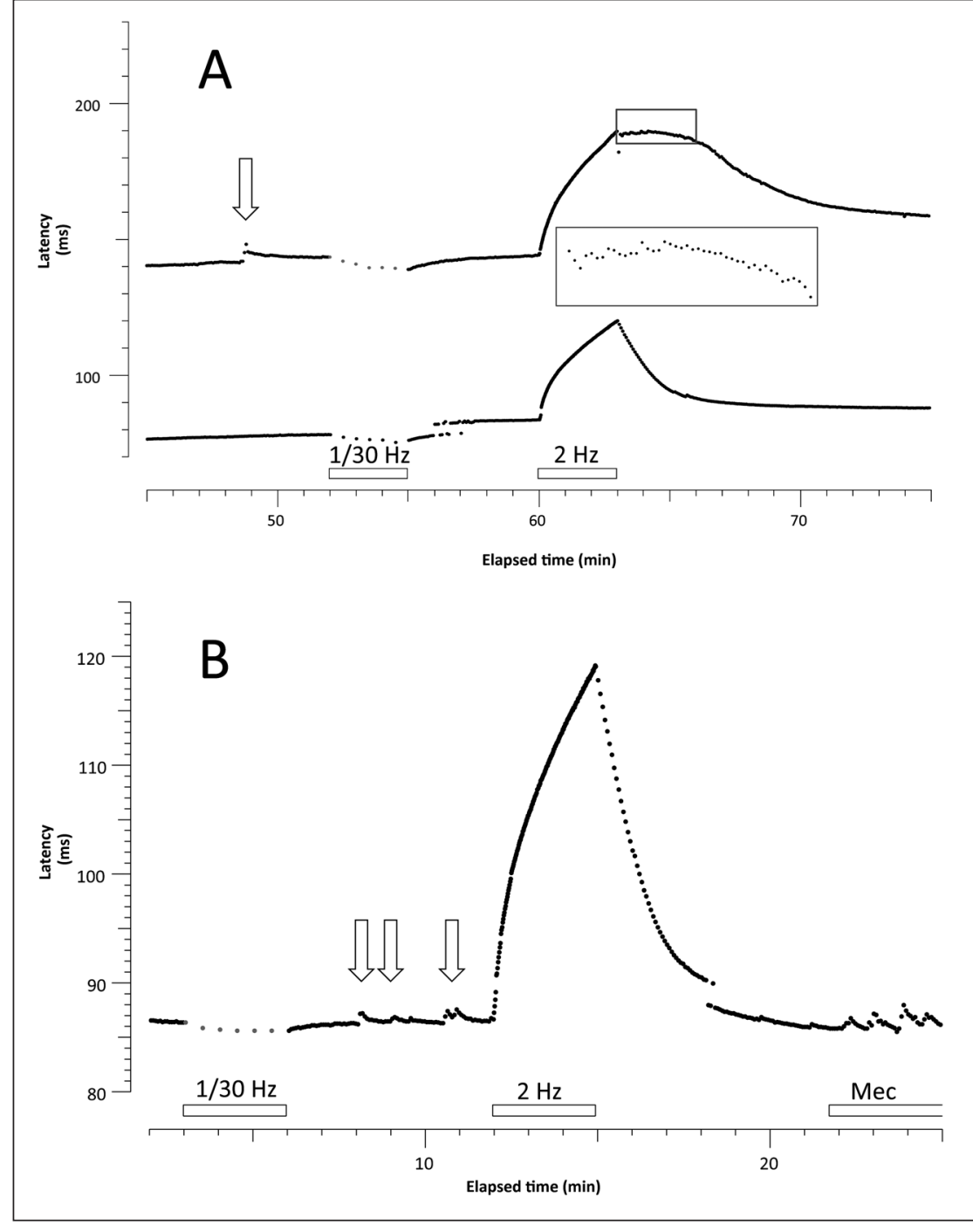

Figura 2. Registro del nervio peroneo superficial de la paciente 1. A. se registran simultáneamente 2 fibras estimulando los campos receptivos con una frecuencia de $0,25 \mathrm{~Hz}$, interrumpido por una 'pausa' de $1 / 30 \mathrm{~Hz}$ y por un 'tétano' de $2 \mathrm{~Hz}$. En el primero la latencia se recupera, mientras que en el segundo se hace más lento mientras dura el tétano. La fibra de latencia más corta corresponde a un nociceptor polimodal, mientras que la más lenta a un nociceptor silente. La flecha indica un súbito aumento de la latencia como consecuencia de una descarga espontánea de esta fibra. La recuperación de la latencia una vez finalizado el tétano es normal en el nociceptor polimodal, mientras que en el nociceptor silente esta recuperación no ocurre sino varios minutos después. Esto porque la fibra se mantiene disparando espontáneamente como se ilustra en el rectángulo ampliado. B. En el mismo paciente se registra de otra fibra con las características de un nociceptor polimodal. Esta fibra responde normalmente al estímulo mecánico (Mec) del campo receptivo, pero también presenta actividad espontánea (flechas). 
su latencia más de $4 \%$ después de la pausa, que corresponden a los nociceptores 'silentes', aquellos que se activan sólo durante la inflamación neurogénica. En este último grupo también estarían los receptores de prurito $^{10}$. En el patrón tipo 2, las fibras aumentan su latencia durante el tétano entre $2-8 \%$ alcanzando una meseta, y corresponden a los termoreceptores de frío ${ }^{11}$. Otro tipo de patrón es el 4, muy similar al tipo 2, pero en estos la latencia cambia rápidamente al inicio del tétano y luego no sólo alcanzan una meseta sino tienden a recuperar la latencia original. Corresponden a eferentes simpáticos cutáneos, vaso o sudomotores $^{8}$. Finalmente, hay un patrón tipo 3, más escaso, que incrementa menos de $3 \%$ la latencia durante el tétano, y corresponden posiblemente a mecanoreceptores de bajo umbral ${ }^{7}$.

Los registros intraneurales se realizaron en el Laboratorio de Neurofisiología en la Facultad de Medicina de la Universidad del Desarrollo, con el consentimiento escrito informado de los pacientes y con la aprobación del comité de Bioética.

\section{Resultados}

\section{Aspectos clínicos}

La Tabla 1 resume los aspectos clínicos de los pacientes. Los 3 individuos presentaban síntomas sensitivos positivos en distribución en calcetín, sugerentes de una polineuropatía. Los síntomas sensitivos incluían hiperalgesia y dolor espontáneo, con un nivel que en ninguno de los pacientes interfería en forma significativa con su funcionamiento diario; en todos, los síntomas eran de predominio nocturno, agravados por el calor y aliviados por la exposición al ambiente frio. En los pacientes 2 y 3 , en los períodos de mayor dolor, la inmersión de los pies en agua fría agregaba un alivio al dolor espontaneo y prurito.
Todos los pacientes tuvieron varias consultas neurológicas con la sospecha de una polineuropatía; el paciente 3 tuvo varias opiniones dermatógicas y múltiples tratamientos tópicos sin resultados beneficiosos. En todos los pacientes los estudios de conducción nerviosa y electromiografía fueron normales, especialmente la amplitud de los potenciales sensitivos de los nervios surales y los reflejos $\mathrm{H}$ en los nervios tibiales, equivalentes electrofisiológicos del reflejo osteotendineosaquilianos (S1).

Los estudios de sensibilidad térmica en los 3 pacientes fueron anormales, mostrando una leve elevación de los umbrales de tibieza en el dorso de los pies (Figura 1). Esta elevación de los umbrales de tibieza contrasta con los umbrales obtenidos en las eminencias tenares y comparado con los resultados de un sujeto normal. En 2/3 pacientes el estímulo frío evocó una sensación caliente paradojal (SCP) con umbrales cercanos a los nociceptivos $\left(\sim 20^{\circ} \mathrm{C}\right)$.

En el paciente 1 y 3 hubo una intolerancia a la glucosa, mientras que en el paciente 2 no se encontraron otras causas de polineuropatía. El estudio también incluyó hemograma, pruebas de función tiroidea, anticuerpos antinucleares y extractables del núcleo, inmunofijación en suero y orina, niveles de vitamina B12, creatinininemia y electrolitos plasmáticos.

\section{Microneurografía}

En los 3 pacientes se registró desde el nervio peroneo superficial a nivel del tobillo sin inconvenientes técnicos. En todos ellos se aisló un fascículo conteniendo fibras $\mathrm{C}$ no mielínicas proyectando hacia la piel de la zona sintomática del dorso distal del pie correspondiente. Los detalles de los hallazgos neurofisiológicos se presentan en la Tabla 2. Se identificaron un total de 21 fibras no mielínicas en los tres pacientes: 8 en la paciente 1 , 7 en el 2 y 6 en el paciente 3 .

Tabla 1.

\begin{tabular}{|ccccccccccccccc|}
\hline Paciente Sexo & Edad & Evol & Parest & Dolor & Prurito & Dist & ROT & Hipo & Hiper & Eritema & Auto & EMG & AST \\
1 & F & 47 & 4 & No & Urente & No & Calcetín & No & No & Sí & Sí & No & NI & Hipo-T/SCP \\
2 & F & 52 & 2 & No & Urente & No & Calcetín & No & No & Sí & Sí & No & NI & Hipo-T \\
3 & M & 37 & 2 & No & urente & Sí & Calcetín & No & No & Sí & Sí & No & NI & Hipo-T/SCP \\
\hline
\end{tabular}

Abreviaciones: Evol= tiempo de evolución (años); Parest= parestesias; dist= ditribución; ROT= reflejos osteo-tendinosos; hipo = hipoestesia; Hiper = hiperalgesia; $A$ uto = síntomas autonómicos; EMG = electromiografía; $A S T=$ análisis de sensibilidad térmica; Hipo-T/SCP = hipoestesia a tibieza con sensación caliente paradojal. 
Actividad espontánea de nociceptores cutáneos en polineuropatías - M. Campero et al

Tabla 2.

\begin{tabular}{|cclcccc|}
\hline Tipo de fibra & $\mathbf{n}$ & V.C. & Pausa \% & $\mathbf{2 ~ H z ~ \%}$ & Activ. Esp & Multipl \\
\hline 1a & 11 & $0,7(0,1)$ & $-0,8(0,7)$ & $26,7(7,2)$ & $4 / 11$ & $0 / 11$ \\
\hline b & 5 & $0,5(0,2)$ & $-2,8(2,2)$ & $41,5(7,0)$ & $3 / 5$ & $0 / 5$ \\
\hline 2 & 1 & 1,1 & $-0,6$ & 5,2 & $n / c$ & $0 / 1$ \\
4 & 3 & $0,6(0,1)$ & 0,4 & $6,5(0,9)$ & $4 / 4$ & $0 / 3$ \\
indef & 1 & 0,4 & $-3,4$ & 24,2 & $1 / 1$ & $1 / 1$ \\
\hline
\end{tabular}

Abreviaciones: V.C. = velocidad de conducción; Activ. Esp= actividad espontánea; Multipl= multiplicación de impulsos; Pausa $\%=$ porcentaje de enlentecimiento durante la pausa; $2 \mathrm{~Hz} \%=$ porcentaje de enlentecimiento durante el tétano.

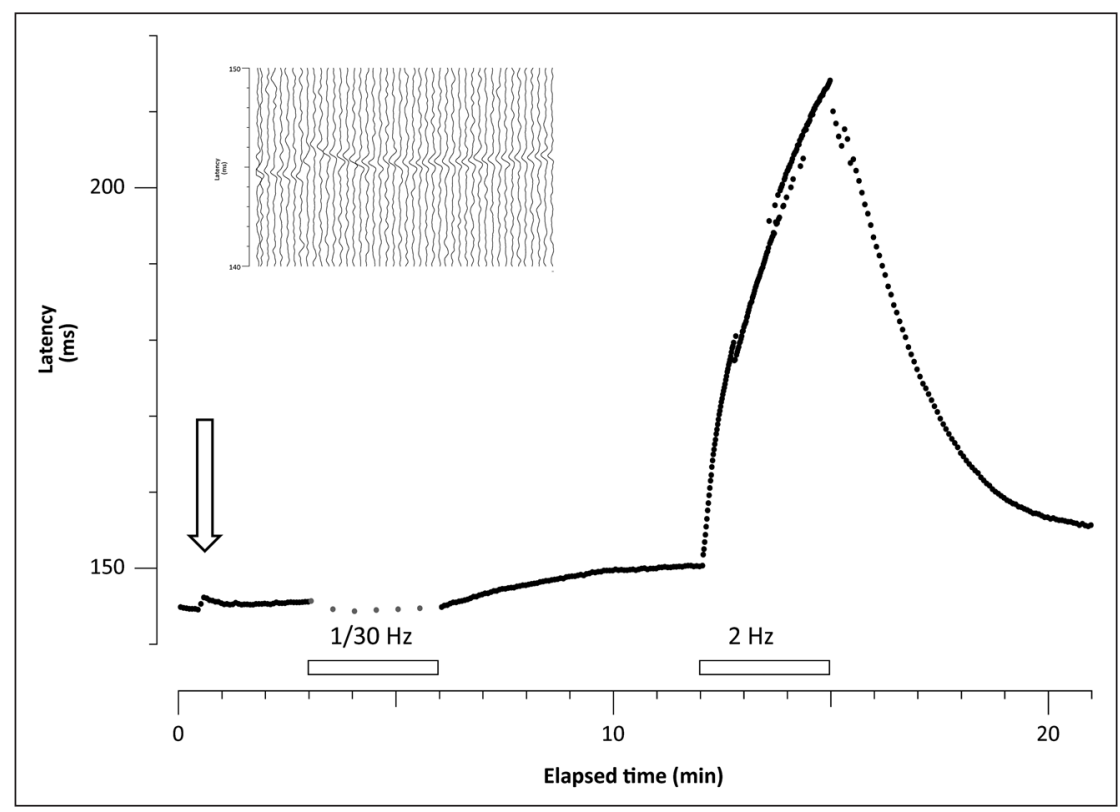

Figura 3. Estimulación repetitiva a $0,25 \mathrm{~Hz}$ de un nociceptor polimodal en paciente 2, interrumpido por una 'pausa' y tétano. Al inicio de la estimulación (flecha) se evidencia un brusco aumento de la latencia en respuesta a una descarga espontánea, recuperando la latencia posteriormente. Esta descarga se muestra con mayor detalle en el inserto superior, en donde se representa el trazo original y no sólo el (los) punto (s) de las respuestas de mayor amplitud. El escaso cambio de latencia durante la 'pausa' demuestra que la fibra corresponde a un nociceptor polimodal.
Se evidenció actividad espontánea en aferentes amielínicos en los tres pacientes. En la paciente 1 se registra actividad espontánea anormal en un aferente con las características de un nociceptor polimodal (1a), así como en un nociceptor silente (1b), como se ilustra en la Figura 2. La actividad espontánea en el nociceptor polimodal tiene similares características a aquella observada durante la estimulación mecánica del campo receptivo con un monofilamento de nylon en rangos nociceptivos $(176 \mathrm{mN})$, como se observa después del tétano de $2 \mathrm{~Hz}$. En el paciente 2, la actividad espontánea ocurre en un aferente con las características de un nociceptor polimodal, como muestra la Figura 3. Al igual que en la paciente 1 , en el paciente 2 la fibra aferente nociceptiva sufre un súbito aumento de la latencia como consecuencia de la generación y propagación de uno, o una salva de potenciales de acción, generados en forma espontánea en algún segmento del axón. Esto es más evidente en la etapa de recuperación después del tétano de $2 \mathrm{~Hz}$ por 3 min, en donde la actividad espontánea es mucho más frecuente (no se ilustra). Finalmente, en el paciente 3, la actividad espontánea se manifiesta en un aferente con las características de un nociceptor silente. La actividad espontánea ocurre durante la estimulación eléctrica de baja frecuencia así como inmediatamente finalizado el tétano de $2 \mathrm{~Hz}$. Esta actividad se ilustra en la Figura 4. En este mismo paciente, un aferente con las características de un nociceptor polimodal, mecanosensible, también presenta actividad espontánea. 

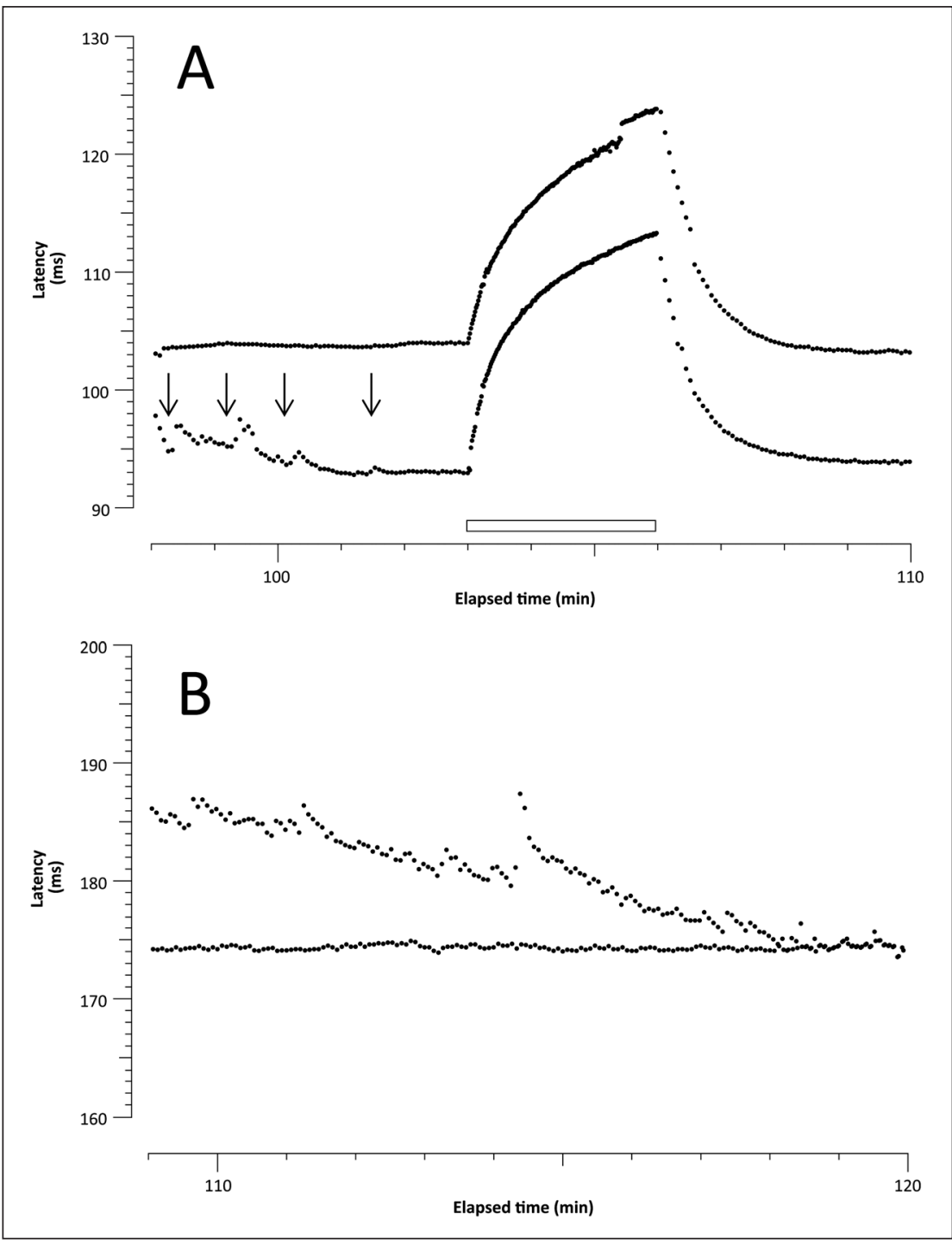

Figura 4. Registro desde el nervio peroneo superficial derecho del paciente 3. A. se registran simultáneamente 2 fibras con las características de nociceptores polimodales. La fibra de menor latencia demuestra fluctuaciones periódicas en su latencia que reflejan una actividad espontanea anormal, posiblemente en salvas de potenciales de acción. En el panel $\mathbf{B}$ se ilustra una fibra de mayor latencia que reduce su latencia en forma progresiva en la medida que disminuye la generación ectópica de impulsos, para alcanzar una latencia similar a la de un nociceptor polimodal que no tiene ningún tipo de actividad anormal. La fibra de mayor latencia corresponde a un nociceptor silente, similar a las fibras de prurito descritas.

\section{Discusión}

Las polineuropatías que afectan sólo, o casi exclusivamente fibras mielínicas delgadas (A delta) y amielínicas C (v.g. 'polineuropatía de fibras delgadas'), tienen habitualmente parámetros clínicos y electrofisiológicos convencionales normales, generando así una dificultad diagnóstica ${ }^{2,12}$. Es el caso de los 3 pacientes de este trabajo en donde el diagnóstico de polineuropatía, si bien se sospechó en todos, no logró confirmarse en ninguno de ellos con los métodos tradicionales. Esto trae como consecuencias retraso en la terapia sintomática y un retardo en estudio causal. De hecho, en los tres pacientes no sólo se plantearon diagnósticos dermatológicos, sin también patologías vasculares periféricas y trastornos de somatización. La presencia de actividad espontánea en aferentes nociceptivos permite documentar la existencia de un proceso patológico de las fibras amielínicas que, por un lado explican los síntomas sensitivos positivos, y por otro, permiten establecer (junto a la elevación de los umbrales térmicos) la existencia una polineuropatía de fibras delgadas. Es interesante destacar que hasta en $28 \%$ de pacientes con polineuropatía de fibras delgadas 'idiopáticas' los 
síntomas son explicados por una mutación del gen que codifica para un canal de sodio dependiente de voltaje ( $\left.\mathrm{Na}_{\mathrm{v}} 1.7\right)$ expresado en aferentes nociceptivos ${ }^{13}$.

La electrofisiología convencional sólo da cuenta del funcionamiento de las fibras mielínicas gruesas, que median la sensación táctil y propioceptiva, y la función motora. Por lo tanto, deben también considerarse métodos que permitan evaluar la función de las fibras delgadas. Dentro de estos métodos está el ATS que evalúa los umbrales de frío y tibieza, así como los de dolor térmico ${ }^{12,14,15}$. Este estudio tiene la desventaja de que requiere de la atención del sujeto para señalar la sensación umbral; sin embargo, son reproducibles y tienen una mínima variabilidad inter e intraobservador ${ }^{16}$. La ventaja es que no sólo evalúa hipofunción (i.e. hipoestesia térmica), sino que también permite evidenciar un exceso de función (i.e. hiperalgesia térmica) que da claves en los mecanismos que subyacen los síntomas sensitivos positivos. En nuestros pacientes, los umbrales de sensibilidad a la tibieza estuvieron elevados (Figura 1), lo que sugiere que la densidad de fibras no mielínicas en la piel está disminuido, compatible con el diagnóstico de una neuropatía de fibras delgadas ${ }^{12}$. La SCP, fenómeno relativamente frecuente en polineuropatía ${ }^{17}$, estuvo presente en $2 / 3$ pacientes. El mecanismo de esta SCP no es del todo claro pero podría corresponder a una sensibilización de nociceptores ${ }^{18} \mathrm{o}$ a la expresión de aferentes sensibles al frío y mentol con pérdida de los aferentes $\mathrm{A}$ delta ${ }^{11}$. Otros métodos diagnósticos de neuropatía de fibras delgadas como los estudios de función autonómica y la biopsia cutánea con cuantificación de la densidad de fibras epidérmicas son complementarios y han sido validadas en múltiples estudios ${ }^{14,19}$.

La microneurografía, desarrollada en Suecia y Alemania a fines de la década 1960-69, es una herramienta de mucho valor en la comprensión de la función de aferentes delgados en sujetos normales ${ }^{5}$, así como en la detección de anomalías funcionales en pacientes con neuropatía doloro$\mathrm{sa}^{20-22}$. Los estudios fisiológicos han permitido inferir el tipo de receptor a partir de las propiedades bioeléctricas del axón: fibras que demuestran un marcado enlentecimiento asociado a la actividad corresponden a nociceptores, mientras que aquellos que se enlentecen en forma moderada son termoreceptores o fibras simpáticas eferentes, mien- tras los que se enlentecen en forma muy discreta son probablemente mecanoreceptores de bajo umbral. Gracias al conocimiento de estas características ha sido posible detectar la presencia de actividad anormal en aferentes amielínicos ${ }^{7-9,11,23}$.

En el presente estudio se reconoce actividad anormal en cerca de 50\% de las unidades aferentes registradas en cada paciente, lo que contrasta con un hallazgo altamente infrecuente en sujetos normales ${ }^{9,24}$. El correlato clínico de actividad espontánea de nociceptores es, con toda probabilidad, el dolor espontaneo y/o prurito ${ }^{10,20}$ mediado por la propagación ortodrómica del potencial de acción hacia el sistema nervioso central, y por otro lado, la inflamación neurogénica -eritralgia- con la invasión antidrómica de potenciales de acción en los terminales de nociceptores cutáneos, particularmente aquellos con las características de nociceptores silentes ${ }^{25,26}$. En nuestros pacientes, el dolor espontaneo es una regla, mientras que el prurito se da preferentemente en uno de ellos. A pesar de ello, el tipo de actividad anormal, o el tipo de fibras que lo manifiesta no es muy diferente. Es posible que el análisis en un grupo grande de pacientes con prurito neurogénico versus pacientes con polineuropatía dolorosa más pura demuestre una diferencia significativa en el tipo de aferente involucrado en la actividad ectópica anormal.

En conclusión, los hallazgos de actividad espontánea en aferentes nociceptivos amielínicos parece un fenómeno frecuente en pacientes con polineuropatía dolorosa. Cuando la manifestación clínica es predominante, o exclusivamente de fibras mielínicas delgadas/amielínicas, los métodos diagnósticos convencionales suelen ser normales, y los estudios cuantitativos somatosensoriales y el registro intraneural, permiten confirmar el diagnóstico de polineuropatía y considerar el estudio etiológico y el tratamiento basado en mecanismos.

\section{Referencias}

1. Low PA, Walsh JC, Huang CY, McLeod JG. The sympathetic nervous system in diabetic neuropathy. A clinical and pathological study. Brain 1975; 98 (3): 341-56.

2. Lacomis D. Small-fiber neuropathy. Muscle Nerve 2002; 26 (2): 173-88.

3. Kimura J. Electrodiagnosis in diseases of nerve and muscle. 3 ed. New York: Oxford University Press; 2001.

4. Verdugo R, Campero M, Cea JG, Castillo JL. Pain and Temperature. In: Goetz CG, Pappert EJ, editors. Text- 
book of Clinical Neurology. 3rd ed. Philadephia: W.B. Saunder; 2007. p. 363-81.

5. Torebjork E, Hallin RG. Skin receptors supplied by unmyelinated (C) fibers in man. In: Zotterman Y, editor. Sensory functions of the skin. Oxford: Pergamon Press; 1976. p. 475-85.

6. Campero M, Serra J, Ochoa JL. C-polymodal nociceptors activated by noxious low temperature in human skin. The Journal of Physiology 1996; 497 ( Pt 2): 565 72 .

7. Campero M, Bostock H, Baumann TK, Ochoa JL. Activity-dependent slowing properties of an unmyelinated low threshold mechanoreceptor in human hairy skin. Neurosci Lett 2011; 493 (3): 92-6.

8. Campero M, Serra J, Bostock H, Ochoa JL. Partial reversal of conduction slowing during repetitive stimulation of single sympathetic efferents in human skin. Acta Physiologica Scandinavica 2004; 182 (3): 305-11.

9. Serra J, Campero M, Ochoa J, Bostock H. Activitydependent slowing of conduction differentiates functional subtypes of $\mathrm{C}$ fibres innervating human skin. The Journal of Physiology 1999; 515 (Pt 3): 799-811.

10. Schmelz M, Schmidt R, Bickel A, Handwerker HO, Torebjork HE. Specific C-receptors for itch in human skin. J Neurosci 1997; 17 (20): 8003-8.

11. Campero M, Baumann TK, Bostock H, Ochoa JL. Human cutaneous $\mathrm{C}$ fibres activated by cooling, heating and menthol. The Journal of Physiology 2009; 587 (Pt 23): 5633-52.

12. Lauria G. Small fibre neuropathies. Curr Opin Neurol 2005; 18 (5): 591-7.

13. Faber CG, Hoeijmakers JG, Ahn HS, Cheng X, Han C, Choi JS, et al. Gain of function Na (V) 1.7 mutations in idiopathic small fiber neuropathy. Ann Neurol 2012; 71 (1): 26-39.

14. England JD, Gronseth GS, Franklin G, Carter GT, Kinsella LJ, Cohen JA, et al. Practice Parameter: evaluation of distal symmetric polyneuropathy: role of autonomic testing, nerve biopsy, and skin biopsy (an evidencebased review). Report of the American Academy of Neurology, American Association of Neuromuscular and Electrodiagnostic Medicine, and American Academy of Physical Medicine and Rehabilitation. Neurology 2009; 72 (2): 177-84.
15. Verdugo R, Ochoa JL. Quantitative somatosensory thermotest. A key method for functional evaluation of small calibre afferent channels. Brain 1992; 115 (Pt 3): 893-913.

16. Arendt-Nielsen L, Yarnitsky D. Experimental and clinical applications of quantitative sensory testing applied to skin, muscles and viscera. J Pain 2009; 10 (6): 556-72.

17. Yarnitsky D, Ochoa JL. Release of cold-induced burning pain by block of cold-specific afferent input. Brain 1990; 113 (Pt 4): 893-902.

18. Serra J, Solà R, Quiles C, Casanova-Molla J, Pascual V, Bostock $\mathrm{H}$, et al. C-nociceptor ssensitized to cold in a patient with small-fiber neuropathy and cold allodynia. Pain 2009; 147: 8.

19. England JD, Gronseth GS, Franklin G, Carter GT, Kinsella LJ, Cohen JA, et al. Evaluation of distal symmetric polyneuropathy: the role of autonomic testing, nerve biopsy, and skin biopsy (an evidence-based review). Muscle Nerve 2009; 39 (1): 106-15.

20. Bostock H, Campero M, Serra J, Ochoa JL. Temperature-dependent double spikes in C-nociceptors of neuropathic pain patients. Brain 2005; 128 (Pt 9): 2154-63.

21. Orstavik K, Namer B, Schmidt R, Schmelz M, Hilliges $\mathrm{M}$, Weidner C, et al. Abnormal function of C-fibers in patients with diabetic neuropathy. J Neurosci 2006; 26 (44): 11287-94.

22. Orstavik K, Weidner C, Schmidt R, Schmelz M, Hilliges $\mathrm{M}$, Jorum E, et al. Pathological C-fibres in patients with a chronic painful condition. Brain 2003; 126 (Pt 3): 567 78 .

23. Schmelz M, Schmid R, Handwerker HO, Torebjork HE. Encoding of burning pain from capsaicin-treated human skin in two categories of unmyelinated nerve fibres. Brain 2000; 123 Pt 3: 560-71.

24. Namer B, Barta B, Orstavik K, Schmidt R, Carr R, Schmelz $\mathrm{M}$, et al. Microneurographic assessment of C-fibre function in aged healthy subjects. The Journal of Physiology 2009; 587 (Pt 2): 419-28.

25. Ochoa JL, Campero M, Serra J, Bostock H. Hyperexcitable polymodal and insensitive nociceptors in painful human neuropathy. Muscle Nerve 2005; 32 (4): 459-72.

26. Serra J, Campero M, Ochoa J. Flare and hyperalgesia after intradermal capsaicin injection in human skin. Journal of Neurophysiology 1998; 80 (6): 2801-10. 